\title{
Development of Mega-electron-volt Ultrafast Electron Diffraction at SLAC National Accelerator Laboratory - Towards a Multifunctional Platform for Ultrafast Science
}

Xiaozhe Shen ${ }^{1}$, Fuhao $\mathrm{Ji}^{1}$, Kozina Michael ${ }^{1}$, Duan Luo ${ }^{2}$, Ming-Fu Lin ${ }^{1}$, Alexander Reid ${ }^{1}$, Stephen Weathersby ${ }^{1}$, Jie Yang ${ }^{1}$ and Xijie Wang ${ }^{1}$

${ }^{1}$ SLAC National Accelerator Laboratory, Menlo Park, California, United States, ${ }^{2}$ Stanford University, Stanford, California, United States

Capturing structural changes of material with atomic spatial and temporal resolutions is of crucial importance to the understanding of the relation between structure and functionality, and the ultimate goal to control energy and matter [1]. For the past decades, ultrafast electron diffraction/microscopy (UED/UEM) has been rapidly developing, aiming to provide the relevant length and time scales for ultrafast science [2]. With the advent of high accelerating gradient radiofrequency photoinjector, high brightness electron beam at mega-electron-volt $(\mathrm{MeV})$ energy has become accessible for UED to reach $\sim 1 \AA$ spatial resolution and $\sim 100$ femtosecond temporal resolution. In 2014, SLAC National Accelerator Laboratory launched a UED/UEM initiative, aiming to provide the world's leading ultrafast electron scattering instrument using MeV electron beams. A prototypical MeV UED beamline was built and has been evolving over the years [3]. Application of $\mathrm{MeV}$ electron beams also provides high flexibility for the beamline to accommodate various scientific samples, covering solid-phase, gas-phase, and liquid-phase sample environments. A variety of pump laser wavelength options are also available, covering the spectrum range from ultraviolet, visible, near-infrared, mid-infrared, to far-infrared. A great number of successful ultrafast scientific results have been achieved at SLAC MeV UED in the regions of condense matter physics, warm-dense matter physics, as well as chemical science [4], which paves the way towards a multifunctional platform for ultrafast science. In 2019, SLAC MeV UED became an official user facility and successfully launched the first user run which was focused on the fields of condense and warm-dense matter physics. The second user run, which is focused on gas-phase experiments, will start from Apri1 2020. In this talk, the multifunctional sample environment platform at SLAC MeV UED, as shown in Fig. 1 , as well as selected ultrafast scientific results from each environment, will be presented. Research and development efforts to further expand the capabilities of SLAC MeV UED will be discussed.
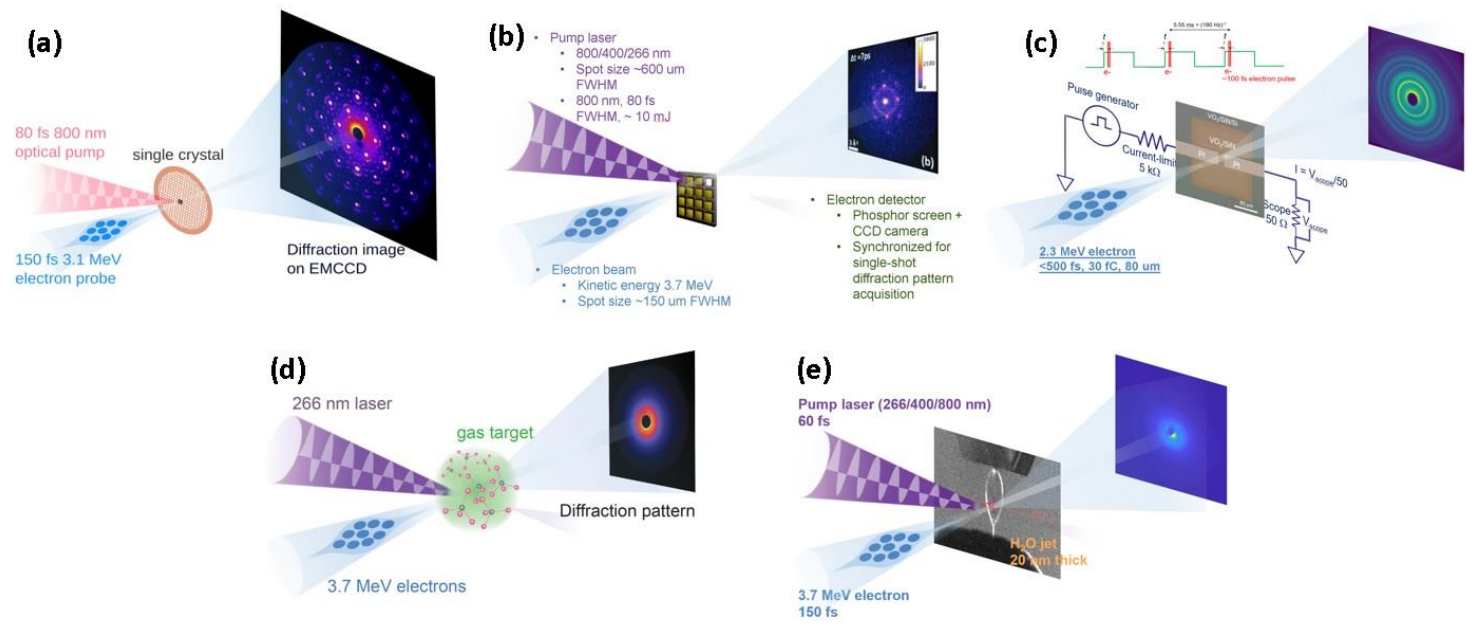
Figure 1. Multifunctional sample environment platform at SLAC MeV UED. (a) condense matter pumpprobe UED. (b) single-shot warm-dense matter pump-probe UED. (c) Electric-field pump condense matter UED. (d) gas-phase pump-probe UED. (e) liquid-phase pump-probe UED.

References

[1] "Directing matter and energy: Five challenges for science and the imagination", A Report from the Basic Energy Sciences Advisory Committee, 2007, (http://science.energy.gov/ /media/bes/pdf/reports/files/gc_rpt.pdf).

[2] R. J. D. Miller, Science 343, 1108 (2014); Ahmed H. Zewail, Science 328, 187 (2010).

[3] S. P. Weathersby, et al., Review of Scientific Instruments 86, 073702 (2015); X. Shen, et al., Ultramicroscopy 2018, 184, 172-176; X. Shen, et al., Struct. Dyn. 6, 054305 (2019).

[4] M. Z. Mo, et al., Science 360, 1451 (2018); J. Yang, et al., Science 361, 64 (2018); E. J. Sie, et al., Nature 565, 61 (2019). 